\title{
Impacto dos sintomas de ansiedade na qualidade de vida na doença de Parkinson: uma revisão sistemática
}

\author{
Impact of anxiety symptoms on quality of life in \\ Parkinson's disease: a systematic review \\ Stephanie Martins de Faria? \\ https://orid.org/0000-0001-8564-3686 \\ Madson Alan Maximiano-Barreto² \\ http://orid.org/0000-0002-1314-9846 \\ Daiene de Morais ${ }^{3}$ \\ https://orcid.org/0000-0001-8609-1190 \\ Marcos Hortes Nisihara Chagas \$, $^{1,3}$ \\ http://orcid.org/0000-0003-3753-7984
}

\section{Palavras-chave}

Ansiedade, qualidade de vida, doença de Parkinson.

\section{Keywords}

Anxiety, quality of life,

Parkinson's disease.

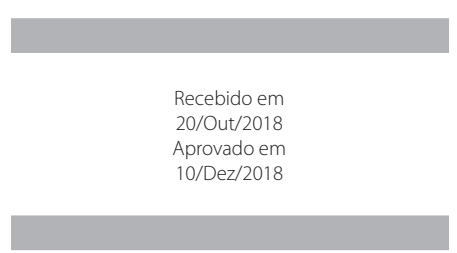

DOI: $10.1590 / 0047-2085000000224$

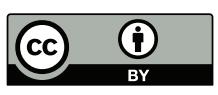

\section{RESUMO}

Objetivo: Revisar estudos relacionados ao impacto da ansiedade na qualidade de vida em pacientes com doença de Parkinson. Métodos: Trata-se de uma revisão sistemática realizada nos bancos de dados PubMed, Scopus, Web of Science e PsycINFO, com os descritores: "anxiety", "Parkinson's disease" e "quality of life". Foram selecionados 20 artigos publicados em inglês e sem limite de tempo. Resultado: A maioria dos estudos demonstrou correlação direta entre ansiedade e qualidade de vida em indivíduos com doença de Parkinson. Apenas dois estudos não encontraram essa associação. A presença de sintomas de ansiedade parece estar relacionada com a gravidade dos sintomas motores, sexo feminino e idade mais jovem, levando a um impacto maior na qualidade de vida. Conclusão: Os artigos analisados mostraram que a ansiedade tem impacto negativo sobre a qualidade de vida de indivíduos com doença de Parkinson.

\section{ABSTRACT}

Objective: To review studies related to the impact of anxiety on the quality of life in Parkinson's disease patients. Methods: A systematic review was performed using PubMed, Web of Science, Scopus and PsycINFO databases with the keywords: anxiety, Parkinson's disease and quality of life. We selected 20 articles published in English, no time limit. Results: Most studies showed positive correlation between anxiety and quality of life in Parkinson's disease individuals. This relation was not found by only two studies. The presence of anxiety symptoms seems to be related to severity of motor symptoms, female sex and early onset of Parkinson's disease, leading to a greater impact on quality of life. Conclusion: The articles analyzed showed that anxiety has negative impact on quality of life of Parkinson's disease individuals.

1 Universidade de São Paulo (USP), Departamento de Neurociências e Ciências do Comportamento, São Paulo, SP, Brasil. 2 Universidade Federal de São Carlos (UFSCar), Departamento de Gerontologia, São Carlos, SP, Brasil. 3 Universidade Federal de São Carlos (UFSCar), Departamento de Psicologia, São Carlos, SP, Brasil.

Endereço para correspondência: Madson Alan Maximiano-Barreto Universidade Federal de São Carlos,

Centro de Ciências Biológicas e da Saúde, Departamento de Gerontologia (DGERO) Rodovia Washington Luís, km 235 13565-905 - São Carlos, SP, Brasil Telefone: (55 82) 99920-8149 E-mail:mmaximianopsi@gmail.com 


\section{INTRODUÇÃO}

A doença de Parkinson (DP) é neurodegenerativa e com alta prevalência em indivíduos com idade igual ou superior aos 60 anos, podendo acometer também indivíduos mais jovens ${ }^{1,2}$. Considera-se a DP como a segunda maior doença neurodegenerativa, com prevalência de até $2 \%$ do total da população com mais de 60 anos $^{3}$. A DP acomete indivíduos de ambos os sexos, no entanto sua prevalência é em indivíduos do sexo masculino $(3: 2)^{4}$.

Essa patologia caracteriza-se por sintomas motores como tremor, rigidez muscular, bradicinesia e instabilidade postural. Essas características são decorrentes da degeneração dos neurônios dopaminérgicos localizados na substância negra 5 . Entre os neurotransmissores, a dopamina é um dos responsáveis pelo controle motor 6 .

A DP, além dos sintomas motores supracitados, apresenta sintomas não motores ${ }^{7,8}$. A ansiedade é um dos seus principais sintomas não motores, com prevalência de 31\% em pacientes com DP8,9. A ansiedade na DP pode ocorrer em consequência da flutuação motora e com maior probabilidade quando os pacientes se encontram no período off ${ }^{10,11}$.

Diversos estudos relacionam a ansiedade com a qualidade de vida (QV), independentemente da presença de comorbidades clínicas ${ }^{11-13}$. Além disso, na DP, os sintomas motores podem piorar na presença de sintomas de ansiedade ${ }^{14}$. Dessa forma, o objetivo deste estudo foi revisar os artigos publicados que avaliaram o impacto da presença de sintomas de ansiedade na QV em indivíduos com DP.

\section{MÉTODOS}

As buscas foram realizadas, até abril de 2018, em quatro bancos de dados: PubMed, Scopus, Web of Science e PsycINFO. As palavras-chave utilizadas foram: "anxiety", "quality of life" e "Parkinson's disease". Os fatores de inclusão foram artigos que avaliaram a relação entre sintomas de ansiedade e QV/bem-estar em uma amostra de pacientes com DP, estudos realizados em humanos, sem limite de tempo e em inglês. Foram excluídos artigos que avaliavam o efeito da ansiedade na QV em pacientes com outras patologias, publicações como editoriais, cartas ao editor, revisões da literatura e artigos com objetivo de validação.

Dois pesquisadores independentes fizeram a extração dos dados e documentaram nome dos autores, ano, local do estudo, desenho do estudo, tamanho da amostra, sexo, idade, critérios utilizados para ansiedade, QV e DP. Os dados foram revistos e qualquer discordância foi discutida entre os autores.

No total, foram encontrados 1.360 artigos. Após a exclusão dos artigos duplicados, 780 artigos foram selecionados. Os resumos deles foram revisados, sendo excluídos 752 arti- gos. Dos 28 artigos selecionados para leitura do texto completo, foram incluídos ao final 20 artigos. De forma detalhada, o processo de busca e seleção dos artigos incluídos nesta revisão sistemática é apresentado na figura 1.

Essa revisão seguiu as orientações da Prisma Statement para revisões sistemáticas da literatura e metanálises de estudos que avaliam intervenções em saúde ${ }^{15}$.

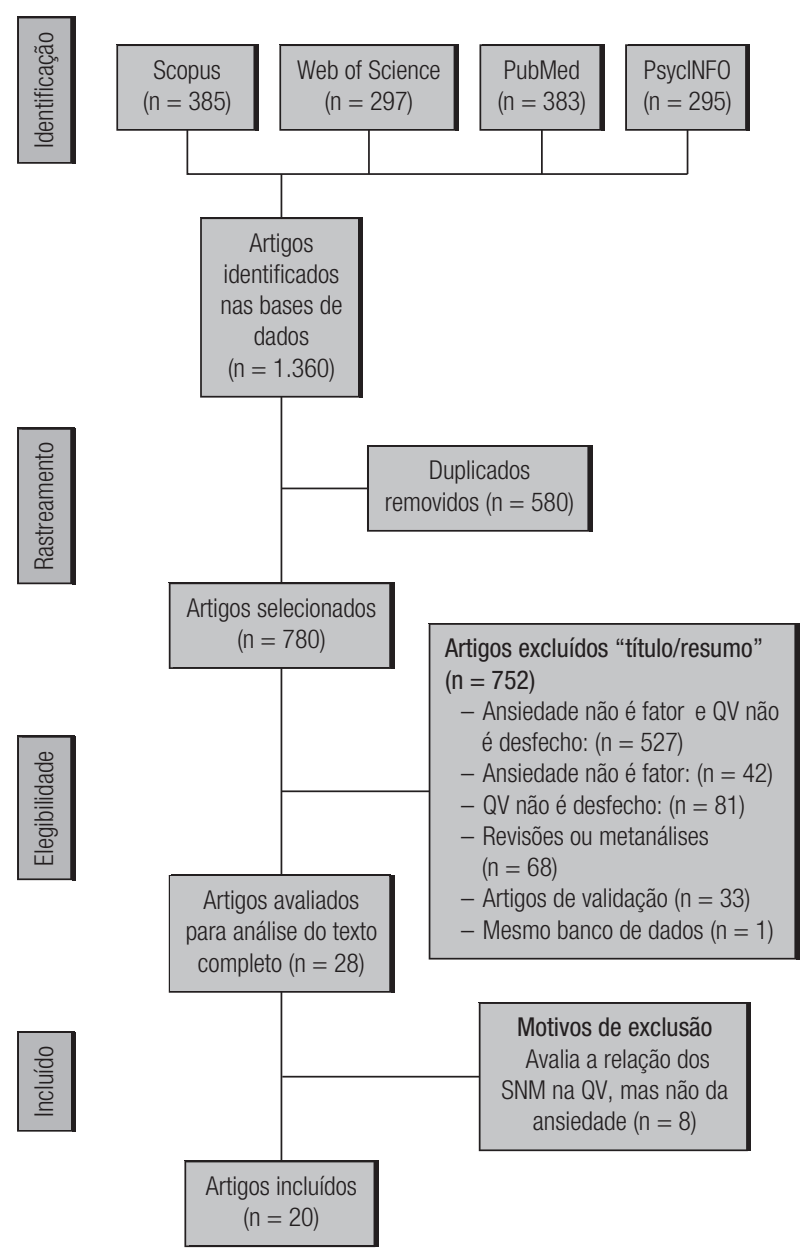

Figura 1. Fluxograma segundo recomendações da iniciativa PRISMA. QV: qualidade de vida, SNM: sintomas não motores. Fonte: Moher D, Liberati A, Tetzlaff J, Altman DG; The PRISMA Group. Preferred Reporting Items for Systematic Reviews and Meta-Analyses: The PRISMA Statement. PLoS Med. 2009;6(6):e1000097..$^{15}$

\section{RESULTADOS}

Dos 1.360 artigos encontrados, 20 foram selecionados com base nos critérios de inclusão e exclusão estabelecidos (Tabela 1).

\section{Características dos estudos selecionados}

Os estudos apresentaram amostras entre 38 e 210 indivíduos $^{16,17}$. Com relação ao perfil dos participantes, $60 \%$ dos estudos foram realizados com pacientes inseridos em clínicas ou hospitais específicos ${ }^{16,18-28}$; quatro estudos recrutaram 
pacientes de universidades ou hospitais universitários 17,29-31; dois estudos foram realizados com indivíduos da comunida$\mathrm{de}^{32,33}$; um estudo foi realizado com pacientes tanto de uma clínica quanto de um grupo de apoio ${ }^{16}$ e outro estudo foi realizado com pacientes inseridos em uma associação de DP34.

A prevalência do sexo masculino variou entre 39,4\% e $72,8 \%$ 9,23. Dois estudos não apresentaram a variável sexo 26,28 . Em relação à idade, a maioria dos estudos apresenta média acima de 60 anos. Park et al. ${ }^{31}$ trabalharam com três grupos: aqueles com DP de início precoce, DP de início médio e DP de início tardio.

Em relação ao desenho dos artigos incluídos, 90,5\% eram transversais $5^{9,16-20,23-32,34,35}$ e apenas dois estudos eram longitudinais 22,33 .

\section{Avaliação de ansiedade}

O instrumento mais utilizado para avaliação da ansiedade foi a Escala Hospitalar de Ansiedade e Depressão (HADS), presente em 28,5\% dos estudos 18,20,24,25,27,32. A Escala de Ansiedade de Beck (BAl) foi utilizada em 19\% dos estudos que avaliaram apenas a ansiedade ${ }^{19,26,30,31}$. Um estudo utilizou essa mesma escala em conjunto com a State-Trait Anxiety Inventory (STAI) ${ }^{16}$ e outro estudo em conjunto com a Hamilton Anxiety Scale (HRSA) ${ }^{34}$. Três estudos utilizaram somente a STAl9,29,33.

\section{Avaliação da qualidade de vida}

Para avaliação da QV, 71,4\% dos estudos utilizaram o instrumento Parkinson's Disease Questionnaire 39 (PDQ-39) 16,19,20,2226,29-32,34,35. Essa mesma escala foi utilizada em conjunto com o Scales for Outcomes in Parkinson's Disease (SCOPAS) ${ }^{18}$. Hanna e Cronin-Golomb ${ }^{16}$ utilizaram o PDQ-39 em conjunto com a Euro-Qol (EQ-5D). Bugalho et al. ${ }^{27}$ utilizaram a EQ-5D isoladamente para avaliar a QV.

Um estudo utilizou a versão reduzida do PDQ-39, o PDQ-8, e um estudo utilizou o instrumento WHOQOL-bref. O PDQ-39 e o SCOPAS são instrumentos específicos para amostras de pacientes com DP 28,33

\section{Associação de ansiedade e qualidade de vida}

Quanto ao desfecho de correlação, em 80,9\% dos estudos foram encontradas correlações significativas entre ansiedade e QV $9,17-20,22-28,30-32,35$. No geral, o valor da correlação variou de 0,22 a $0,75^{9,16,20}$.

Para estabelecimento do nível de correlação, foi considerada correlação fraca para $r<30$, moderada entre 0,30 e 0,59 e forte para $\geq 60$. A análise de regressão multivariada foi utilizada para avaliação dos preditores de QV. Os valores " $\beta$ " se referem à variação que a ansiedade tem sobre a QV. Nos estudos que usaram essa técnica o valor de " $\beta$ " foi de 0,156 a 0,72022,31.

Para estudos que tinham como objetivo avaliar o impacto dos sintomas não motores na QV (inclusive a ansiedade), as correlações de ansiedade e QV variaram de
0,31 a 0,75 17,20,26,27,29,35 e os valores de predição, de 0,156 a $0,699,18,22,24,25,32$. É importante considerar que a utilização de diferentes instrumentos na mensuração da QV influenciou na diferenciação dos valores de correlação, já que os resultados de instrumentos específicos de QV para amostras de pacientes com DP mostraram correlações maiores.

Dois estudos mostraram que a ansiedade não impacta a QV em pacientes com DP. Hurtado-Gonzalez et al. ${ }^{34}$ não obtiveram correlação significativa, da mesma forma Prasuhn et al.33 apresentaram que não houve correlação entre STAI e WHOQOL-bref.

\section{DISCUSSÃO}

Diante da análise integral dos artigos, a maioria dos resultados encontrados nessa revisão mostrou que a ansiedade tem impacto negativo sobre a QV em pacientes com DP e algumas variáveis poderiam ter influenciado esse desfecho.

Considerando os aspectos demográficos, evidenciou-se uma maior prevalência de ansiedade nas mulheres com DP9,17,20,26. Essa evidência é atestada, já que diante dos critérios para diagnóstico de ansiedade, o sexo feminino é considerado um dos fatores de risco para a presença de ansiedade ${ }^{36,37}$. Além disso, num recente estudo de Broen et al. ${ }^{13}$ utilizando um instrumento específico para avaliação da ansiedade na DP, o Parkinson Anxiety Scale (PAS), o sexo feminino foi considerado um importante fator associado à ansiedade persistente e episódica ${ }^{13}$. Uma maior prevalência de sintomas de ansiedade é encontrada em diversos estudos, independente da presença de DP, devido principalmente ao papel dos hormônios sexuais ${ }^{38}$, porém não devem ser descartados aspectos socioeconômicos, e maior exposição a traumas precoces.

Outro fator relevante é o diagnóstico precoce de DP. O estudo de Fereshtehnejad et al. ${ }^{24}$ mostrou que a presença de ansiedade foi maior nos parkinsonianos com idade abaixo dos 50 anos. Corroborando com resultados encontrados nesse estudo, uma pesquisa desenvolvida por Moriyama et al. ${ }^{39} \mathrm{com}$ ansiedade social apresentou o mesmo resultado em indivíduos com DP abaixo dos 50 anos. Dissanayaka et al..$^{40}$ também verificaram que pacientes com DP com início precoce eram mais propensos a experimentar a ansiedade se comparada à DP tardia. De forma similar, Henderson et al. ${ }^{41}$ obtiveram que o início da DP antes dos 60 anos esteve associado com aumento da prevalência dos sintomas de ansiedade. Além disso, Quelhas e $\operatorname{Costa}^{20}$ não encontraram associação significativa entre a ansiedade e o aumento da idade ao avaliar 43 pacientes com DP idiopática atendidos em um ambulatório de distúrbio do movimento. A maior prevalência de ansiedade em indivíduos mais jovens com DP pode estar relacionada ao estresse psicossocial enfrentado por estes indivíduos ${ }^{39}$, prejudicando a QV. 


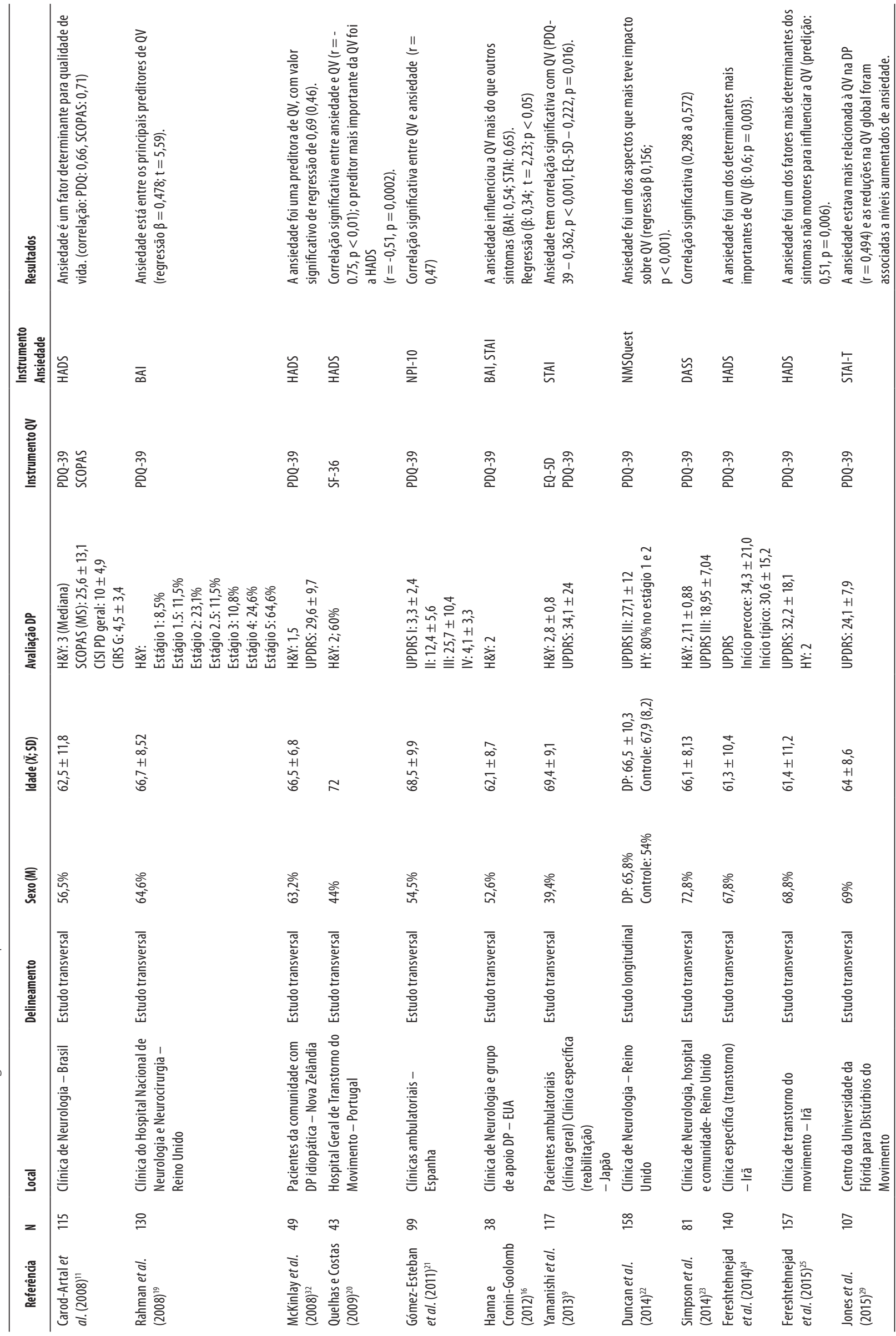




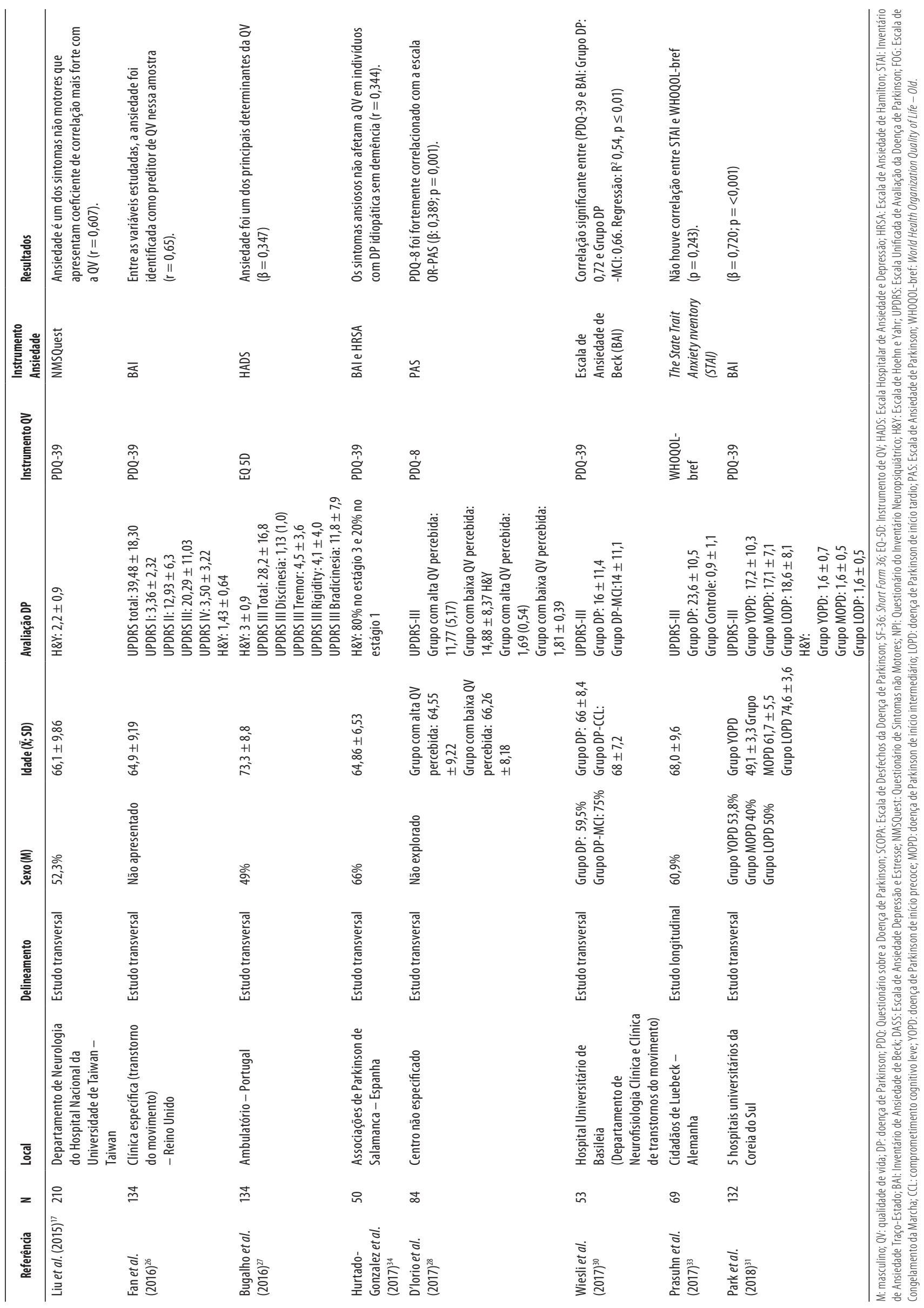

J Bras Psiquiatr. 2019;68(1):48-55 
O tamanho amostral é outro importante ponto de discussão, uma vez que os estudos de Hurtado-Gonzalez et al. ${ }^{34}$ e Prasuhn et al. ${ }^{33}$ foram os únicos que encontraram que a ansiedade não influencia a QV, e a amostra foi composta por menos que 70 indivíduos em ambos os estudos. No entanto, deve-se considerar que outros fatores podem ter influenciado esse desfecho, visto que outras pesquisas com número da amostra pequena apresentaram relação significativa da ansiedade e QV ${ }^{16,20,32}$. Por exemplo, o estudo de Prasuhn et al. ${ }^{33}$ utilizou em sua análise todos os sujeitos, incluídos os com sinais parkinsonianos leves e sem DP, o que pode ter influenciado os resultados. Outra possibilidade que deve ser aventada é que os sintomas de ansiedade podem ser sobrepostos a outros sintomas não motores como depressão, o que minimizaria o impacto da ansiedade na QV ${ }^{34}$

No estudo de McKinlay et al.32, o controle dos sintomas motores foi um aspecto que contribuiu para o impacto da ansiedade na QV. Corroborando essa interpretação, Hurtado-Gonzalez et al. ${ }^{34}$ destacam que a sobreposição dos sintomas motores foi uma das variáveis que influenciou seu desfecho. Da mesma forma, a presença de sintomas motores como a bradicinesia, distonia e discinesia também se mostrou preditiva de altas taxas de ansiedade $37,39,42,43$. Ademais, flutuações nos sintomas de ansiedade podem ocorrer paralelamente às flutuações motoras ${ }^{44}$, o que tornaria a análise do impacto da ansiedade ainda mais difícil, visto que a percepção de QV também poderia mudar de acordo com os níveis de ansiedade.

Foi observado que diversos estudos não explicitam de forma clara os critérios de exclusões, o que pode dificultar a interpretação dos resultados 17,19,21,22,23,25,27. No estudo de Gómez-Esteban et al..21, não foram excluídas pessoas com transtornos neurocognitivos ou demência e foi encontrada correlação significativa entre ansiedade e QV. Todavia, a presença de demência pode ser um fator importante na associação entre QV e ansiedade mesmo em pacientes sem DP45. Além disso, tanto o estado de ansiedade quanto o traço de ansiedade podem influenciar no desempenho cognitivo ${ }^{46}$, introduzindo uma nova variável na relação já complexa entre ansiedade de QV.

A utilização de diferentes instrumentos também pode ter contribuído para distintos resultados de correlação. Para estudos que utilizaram o PDQ-39 concomitantemente a um instrumento não específico para QV na DP, como o SF-36 e o EQ-5D, foram observadas correlações menores para esses instrumentos de QV não específicos ${ }^{9,18}$. Além disso, o estudo de Prasuhn et al. ${ }^{33}$ não apresentou correlação da ansiedade com a QV, e uma das possíveis explicações seria a não utilização de um instrumento específico para avaliação da QV, considerando aspectos específicos da DP ${ }^{33}$. Esse ponto é relevante, visto que instrumentos específicos consideram aspectos peculiares à DP, fornecendo um retrato mais fidedigno do impacto na QV. Por exemplo, no PDQ-39, existem questões que abordam especificamente o constrangimento em público e as reações das pessoas em relação à DP, o que não é mensurado por outras escalas de QV.

Para avaliação de ansiedade, diferentes instrumentos foram utilizados; desses, apenas BAl, STAI e HRSA são exclusivos para o rastreio de ansiedade, porém não consideram as peculiaridades da DP. Dessa forma, insere-se a necessidade de utilização de instrumentos adaptados para o contexto do paciente com DP, como o PAS, que superaria as limitações das escalas existentes, considerando em suas subescalas aspectos como a ansiedade persistente, episódica e o comportamento de evitação ${ }^{47}$. Nessa revisão, encontramos um único estudo que utilizou o PAS para avaliação da ansiedade, mostrando que baixos níveis de QV apresentaram ansiedade episódica, persistente e comportamento de evitação mais graves do que pacientes com QV alta ${ }^{28}$. A utilização de uma escala específica e padronizada poderia facilitar a comparação entre os diversos estudos encontrados nesta revisão.

Como limitação, apresenta-se que poucos estudos verificaram a correlação dos sintomas motores e não motores com a ansiedade, visto que a ansiedade pode estar frequentemente associada à presença e à gravidade de sintomas motores ${ }^{9,20,26,27,32}$. Além disso, seria interessante avaliar o impacto de transtornos de ansiedade específicos na QV, visto que a apresentação dos sintomas de ansiedade é diferente para cada um deles.

\section{CONCLUSÃO}

Nesta revisão, os artigos analisados mostraram que a ansiedade piora a QV de indivíduos com DP. Estudos futuros devem considerar a utilização de escalas capazes de identificar as peculiaridades da DP para a eficaz avaliação da ansiedade. Para isso, faz-se necessária a utilização de instrumentos que considerem a influência de aspectos característicos da DP na ansiedade. Além disso, seriam importantes estudos para avaliar o impacto de transtornos de ansiedade específicos na DP. Os sintomas motores parecem ter impacto importante na ansiedade e QV, porém deve-se explorar mais detalhadamente a relação bidirecional entre ansiedade e sintomas motores na DP. Compreender esses fatores auxiliará no manejo do tratamento da ansiedade nos pacientes com DP.

Essas pesquisas são importantes, pois podem contribuir para a prática clínica no tratamento da DP, considerando conjuntamente os sintomas clássicos da doença, o diagnóstico e o tratamento da ansiedade para a promoção do bem-estar e QV.

\section{CONTRIBUIÇÕES INDIVIDUAIS}

Stephanie Martins de Faria - Escreveu a primeira versão da revisão e realizou a revisão dos artigos e a finalização do manuscrito. 
Madson Alan Maximiano-Barreto - Contribuiu na escrita, revisão dos artigos e busca dos artigos.

Daiene de Moraes - Contribuiu na escrita, revisão dos artigos e busca dos artigos.

Marcos Hortes N. Chagas - Supervisionou a busca de artigos e contribuiu na escrita e finalização do manuscrito.

\section{CONFLITOS DE INTERESSE}

Todos os autores declararam não apresentar conflitos de interesse.

\section{AGRADECIMENTOS}

O presente trabalho foi realizado com o apoio da Coordenação de Aperfeiçoamento de Pessoal de Nível Superior - Brasil (Capes) - Código de Financiamento 001, Fundação de Amparo à Pesquisa do Estado de São Paulo - Fapesp (2016/06561-2) e Conselho Nacional de Desenvolvimento Científico e Tecnológico - Brasil (CNPq) - processo no 132257/2017-3.

\section{REFERÊNCIAS}

1. Ferraz HB, Borges V. Doença de Parkinson. Rev Bras Med. 2002;59(4):207-19.

2. Sprenger F, Poewe W. Management of motor and non-motor symptoms in Parkinson's disease. CNS Drugs. 2013;27(4):259-72.

3. Rijk MD, Tzourio C, Breteler MM, Dartigues JF, Amaducci L, Lopez-Pousa S, et al. Prevalence of parkinsonism and Parkinson's disease in Europe: the EUROPARKINSON Collaborative Study. European Community Concerted Action on the Epidemiology of Parkinson's disease. J Neurol Neurosurg Psychiatry. 1997;62(1):10-5

4. Beitz JM. Parkinson's disease: a review. Front Biosci. 2014;6(1):65-74

5. Maiti P, Manna J, Dunbar GL. Current understanding of the molecular mechanisms in Parkinson's disease: Targets for potential treatments. Transl Neurodegener. 2017;6(1):1-35.

6. Azevedo MFA, Meyer A. [Essential tremor in endemic disease control agents exposed to pesticides: a case-control study]. Cad Saude Publica. 2017;33(8):1-12.

7. Kovács M, Makkos A, Weintraut R, Karádi K, Janszky J, Kovács N. Prevalence of Anxiety among Hungarian Subjects with Parkinson's Disease. Behav Neurol. 2017;2017:1470149.

8. Broen MP, Narayen NE, Kuijf ML, Dissanayaka NN, Leentjens AF. Prevalence of anxiety in Parkinson's disease: A systematic review and meta-analysis. Mov Disord. 2016;31(8):1125-33.

9. Yamanishi T, Tachibana H, Oguru M, Matsui K, Toda, K, Okuda B, et al. Anxiety and depression in patients with Parkinson's disease. Intern Med. 2013;52(5):539-45.

10. Witjas T, Kaphan E, Azulay JP, Blin 0, Ceccaldi M, Pouget J, et al. Nonmotor fluctuations in Parkinson's disease: frequent and disabling. Neurology. 2002;59(3):408-13.

11. Carod-Artal FJ, Ziomkowski S, Mourão Mesquita H, Martínez-Martin P. Anxiety and depression: main determinants of health-related quality of life in Brazilian patients with Parkinson's disease. Parkinsonism Relat Disord. 2008;14(2):102-8.

12. Barone P, Antonini A, Colosimo C, Marconi R, Morgante L, Avarello TP, et al. The PRIAMO study: a multicenter assessment of nonmotor symptoms and their impact on quality of life in Parkinson's disease. Mov Disord. 2009;24 (11):1641-9.

13. Broen MP, Leentjens AFG, Hinkle JT, Moonen AJH, Kuijf ML, Fischer NM, et al. Clinical Markers of Anxiety Subtypes in Parkinson Disease. J Geriatr Psychiatry Neurol. 2018;31(2):55-
14. Chagas MHN, Oliveira THG, Linares IM, Balarini FB, Chagas NMS, Tumas V, et al. Can anxiety increase tremors in patients with Parkinson's disease? An experimental model. Arch Clin Psychiatry (São Paulo). 2017;44(4):85-8.

15. Moher D, Liberati A, Tetzlaff J, Altman DG; The PRISMA Group (2009). Preferred Reporting Items for Systematic Reviews and Meta-Analyses: The PRISMA Statement. PLoS Med 6(6): e1000097. doi:10.1371/journal.pmed1000097.

16. Hanna KK, Cronin-Golomb A. Impact of anxiety on quality of life in Parkinson's disease. Parkinson's Dis. 2012;2012:640707.

17. Liu WM, Lin RJ, Yu RL, Tai CH, Lin CH, Wu RM. The impact of nonmotor symptoms on quality of life in patients with Parkinson's disease in Taiwan. Neuropsychiatr Dis Treat. 2015;11:2865-73.

18. Carod-Artal FJ, Vargas AP, Martinez-Martin P. Determinants of quality of life in Brazilian patients with Parkinson's disease. Mov Disord. 2008;22(10):1408-15.

19. Rahman S, Griffin HJ, Quinn NP, Jahanshahi M. Quality of life in Parkinson's disease: the relative importance of the symptoms. Mov Disord. 2008;23(10):1428-34

20. Quelhas R, Costa M. Anxiety, depression, and quality of life in Parkinson's disease. J Neuropsychiat Clin Neurosci. 2009;21(4):413-9.

21. Gómez-Esteban JC, Tijero B, Somme J, Ciordia R, Berganzo K, Rouco I, et al. Impact of psychiatric symptoms and sleep disorders on the quality of life of patients with Parkinson's disease. J Neurol. 2011;258(3):494-9.

22. Duncan GW, Khoo TK, Yarnall AJ, O'Brien JT, Coleman SY, Brooks DJ, et al. Health-related quality of life in early Parkinson's disease: The impact of nonmotor symptoms. Mov Disord. 2014;29(2):195-202.

23. Simpson J, Lekwuwa G, Crawford T. Predictors of quality of life in people with Parkinson's disease: evidence for both domain specific and general relationships. Disab Rehabil. 2014;36(23):1964-70.

24. Fereshtehnejad SM, Hadizadeh H, Farhadi F, Shahidi GA, Delbari A, Lökk J. Comparison of the psychological symptoms and disease-specific quality of life between early-and typical-onset Parkinson's disease patients. Parkinson's Dis. 2014;2014:819260.

25. Fereshtehnejad SM, Shafieesabet M, Farhadi F, Hadizadeh H, Rahmani A, Naderi N, et al Heterogeneous determinants of quality of life in different phenotypes of Parkinson's disease. PloS One. 2015;10(9):e0137081

26. Fan JY, Chang BL, Wu YR. Relationships among depression, anxiety, sleep, and quality of life in patients with Parkinson's disease in Taiwan. Parkinson's Dis. 2016;2016:4040185.

27. Bugalho P, Lampreia T, Miguel R, Mendonça MD, Caetano A, Barbosa R. Non-Motor symptoms in Portuguese Parkinson's Disease patients: correlation and impact on Quality of Life and Activities of Daily Living. Sci Rep. 2016:6:32267.

28. D'Iorio A, Vitale C, Piscopo F, Baiano C, Falanga AP, Longo K, et al. Impact of anxiety, apathy and reduced functional autonomy on perceived quality of life in Parkinson's disease. Parkinsonism Relat Disord. 2017:43:114-7.

29. Jones JD, Butterfield LC, Song W, Lafo J, Mangal P, Okun MS, et al. Anxiety and depression are better correlates of Parkinson's disease quality of life than apathy. J Neuropsychiatry Clin Neurosci. 2015;27(3):213-8.

30. Wiesli D, Meyer A, Fuhr P, Gschwandtner U. Influence of Mild Cognitive Impairment, Depression, and Anxiety on the Quality of Life of Patients with Parkinson Disease. Dement Geriatr Cogn Dis Extra. 2017;7(3):297-308

31. Park HR, Youn J, Cho JW, Oh ES, Kim JS, Park S, et al. Characteristic Motor and Nonmotor Symptoms Related to Quality of Life in Drug-Naïve Patients with Late-Onset Parkinson Disease. Neurodegener Dis. 2018;18(1):19-25

32. McKinlay A, Grace RC, Dalrymple-Alford JC, Anderson T, Fink J, Roger D. A profile of neuropsychiatric problems and their relationship to quality of life for Parkinson's disease patients without dementia. Parkinsonism Relat Disord. 2008;14(1):37-42.

33. Prasuhn J, Piskol L, Vollstedt EJ, Graf J, Schmidt A, Tadic V, et al. Non-motor symptoms and quality of life in subjects with mild parkinsonian signs. Acta Neurol Scand. 2017;136(5):495-500

34. Hurtado-Gonzalez CA, Ladera V, Perea MV, Garcia R. Influence of anxiety symptoms on quality of life in idiopathic Parkinson's disease without dementia. Biomed Res. 2017;28(4):1727-32.

35. Kinrys G, Wygant LE. Anxiety disorders in women: does gender matter to treatment?. Rev Bras Psiquiatr. 2005;27 Suppl 2:s43-550. 
36. Remes 0 , Brayne C, van der Linde R, Lafortune L. A systematic review of reviews on the prevalence of anxiety disorders in adult populations. Brain Behav. 2016;6(7):1-33.

37. Cui SS, Du JJ, Fu R, Lin YQ, Huang P, He YC, et al. Prevalence and risk factors for depression and anxiety in Chinese patients with Parkinson disease. BMC Geriatr. 2017;17:270.

38. Li SH, Graham BM. Why are women so vulnerable to anxiety, trauma-related and stressrelated disorders? The potential role of sex hormones. Lancet Psychiatry. 2017;4(1):73-82.

39. Moriyama TS, Chagas MHN, Silveira-Moriyama L, Tumas, V, Lees AJ, Crippa JA, et al. Diagnosing social anxiety in Parkinson's disease: characteristics and frequencies according to two diagnostic criteria. Arch Clin Psychiatry (São Paulo). 2016;43(6):139-42.

40. Dissanayaka NN, Sellbach A, Matheson S, O'Sullivan JD, Silburn PA, Byrne GJ, et al. Anxiety disorders in Parkinson's disease: prevalence and risk factors. Mov Disord. 2010;25(7):838-45.

41. Henderson R, Kurlan R, Kersun JM, Como P. Preliminary examination of the comorbidity of anxiety and depression in Parkinson's disease. J Neuropsychiatry Clin Neurosci. 1992;4(3):257-64
42. Bolluk B, Özel-Kizil ET, Akbostanci MC, Atbasoglu EC. Social anxiety in patients with Parkinson's disease. J Neuropsychiatry Clin Neurosci. 2010;22(4):390-4.

43. Pontone GM. Anxiety in Parkinson's: a complex syndrome of nondopaminergic and dopaminergic etiology. Eur J Neurol. 2017;24(4):541-2.

44. van der Velden RMJ, Broen MPG, Kuijf ML, Leentjens AFG. Frequency of mood and anxiety fluctuations in Parkinson's disease patients with motor fluctuations: A systematic review. Mov Disord. 2018;33(10):1521-7.

45. Goyal AR, Bergh S, Engedal K, Kirkevold M, Kirkevold $\emptyset$. Trajectories of quality of life and their association with anxiety in people with dementia in nursing homes: A 12-month follow up study. PLoS One. 2018;13(9):e0203773.

46. Ehgoetz Martens KA, Silveira CRA, Intzandt BN, Almeida QJ. State anxiety predicts cognitive performance in patients with Parkinson's disease. Neuropsychology. 2018;32(8):950-957.

47. Leentjens AF, Dujardin K, Pontone GM, Starkstein SE, Weintraub D, Martinez-Martin P. The Parkinson Anxiety Scale (PAS): development and validation of a new anxiety scale. Mov Disord. 2014;29(8):1035-43 